\title{
Dominant Large-Scale Atmospheric Circulation Systems for the Extreme Precipitation over the Western Sichuan Basin in Summer 2013
}

\author{
Yamin Hu, ${ }^{1,2}$ Panmao Zhai, ${ }^{2}$ Lihong Liu, ${ }^{2}$ Yang Chen, ${ }^{2}$ and Yanju Liu ${ }^{3}$ \\ ${ }^{1}$ Guangdong Climate Center, Guangzhou 510080, China \\ ${ }^{2}$ State Key Laboratory of Severe Weather, Chinese Academy of Meteorological Sciences, Beijing 100081, China \\ ${ }^{3}$ National Climate Center, Beijing 100081, China
}

Correspondence should be addressed to Panmao Zhai; pmzhai@cma.gov.cn

Received 21 October 2014; Accepted 3 December 2014

Academic Editor: Jieshun Zhu

Copyright (C) 2015 Yamin Hu et al. This is an open access article distributed under the Creative Commons Attribution License, which permits unrestricted use, distribution, and reproduction in any medium, provided the original work is properly cited.

\begin{abstract}
The western Sichuan Basin (WSB) is a rainstorm center influenced by complicated factors such as topography and circulation. Based on multivariable empirical orthogonal function technique for extreme precipitation processes (EPP) in WSB in 2013, this study reveals the dominant circulation patterns. Results indicate that the leading modes are characterized by "Saddle" and "Sandwich" structures, respectively. In one mode, a TC from the South China Sea (SCS) converts into the inverted trough and steers warm moist airflow northward into the WSB. At the same time, WPSH extends westward over the Yangtze River and conveys a southeasterly warm humid flow. In the other case, WPSH is pushed westward by TC in the Western Pacific and then merges with an anomalous anticyclone over SCS. The anomalous anticyclone and WPSH form a conjunction belt and convey the warm moist southwesterly airflow to meet with the cold flow over the WSB. The configurations of WPSH and TC in the tropic and the blocking and trough in the midhigh latitudes play important roles during the EPPs over the WSB. The persistence of EPPs depends on the long-lived large-scale circulation configuration steady over the suitable positions.
\end{abstract}

\section{Introduction}

A particular challenge to operational weather forecasters is extreme precipitation processes (EPPs) forecasting, and especially those topography-related events are more complicated, such as over the Taiwan [1-3], the Mediterranean [4], the Rocky Mountains [5].

The western Sichuan Basin (WSB) $\left(102^{\circ}-108^{\circ} \mathrm{E}, 28^{\circ}-33^{\circ} \mathrm{N}\right)$ is well-known as a rainy region in the western China, which is also one of rainstorm centers in China [6]. Some studies concentrated the effects of the topography of the Sichuan Basin and the Tibetan Plateau on the precipitation events by numerical simulation and observation analyses [7-11].

Numerous studies paid more attention to the favorable weather systems for the formation and maintenance of heavy rainfall over the WSB, such as the mid-high latitude blocking and trough, the Western Pacific Subtropical High (WPSH) [12-15], the southwest vortex [16-18], the South Asia high
[19], and other mesoscale synoptic systems [20]. Shi et al. [14] pointed out that $84 \%$ of rainstorm processes could be associated with the WPSH during June-September from 1998 to 2008 over Sichuan. However, most rainstorm processes are influenced by the different position of the WPSH [21]. During the rainy season (May 1 to September 30), four typical circulation patterns were summarized over the WSB [22], including the plateau vortex pattern, the shortwave trough pattern in the westerlies, one trough between two ridge patterns and the shear line pattern over the Yangtze-Huaihe region. More interestingly, both the circulation systems of one trough between the double blocking highs $[13,22,23]$ and those of one ridge between the double troughs [24-26] could be beneficial to the production of heavy precipitation over WSB. Why could the seemingly contradictory or different configurations be favorable for heavy rainfall over WSB?

Furthermore, the above mentioned researches mainly focus on the importance of the mid-high latitude circulation 
systems. Chen et al. [27] addressed that the depression in the tropic also played an important role in EPPs over Sichuan on July 26-30, 2001, July 30-August 1, 2002, and September 23-26, 2008. Few studies of EPPs over WSB, however, gave enough consideration to the circulation systems from the low latitudes and the circulation configurations from the different latitude.

Recently, more attentions have been paid to EPPs because they have the potential to become hazardous virtually anywhere in the world. Doswell et al. [4] investigated three cases of project ANOMALIA with heavy precipitation in the western Mediterranean region. Their evaluation showed the unique characteristics of each event, as well as some limited similarities.

During summer 2013, WSB was frequently attacked by EPPs, especially the earthquake-hit areas (i.e., Wenchuan and Yaan). Extreme precipitations resulted in serious natural disasters, like mountain torrents and landslides. The severe floods and their associated natural disasters caused lots of human life and property damage as well. There were 358 large-scale geological disasters. The unusually heavy rain caused flash floods in the Minjiang River, the Tuojiang River, and so on. In addition, statistics data reported that 16 million people were affected by the flood which also caused direct economic loss of 41.5 billion Yuan (RMB). That catastrophe has undoubtedly intensified the disaster over geologically vulnerable regions such as Wenchuan, Yaan, and other earthquake-hit areas. However, what are the dominant circulation systems and how do they match up to result in the EPPs over WSB during summer 2013? What are the similarities among the circulation systems during the EPPs?

In this study, we will focus on the above questions. Firstly, by exploring the daily anomalous circulation of the $500-\mathrm{hPa}$ geopotential height and the water vapor transport features, the main impacting circulation systems are analyzed. Further, by applying the multivariable empirical orthogonal function (MV-EOF) technique, two dominant patterns and their corresponding atmospheric circulation configurations, as well as their conceptual models, are demonstrated.

The rest of this paper is organized as follows. Descriptions of data and analysis method are given in Section 2. The extreme precipitation processes overviews are discussed in Section 3. In Section 4, large-scale circulation and water vapor transport features for each process are investigated, so as to capture their impacting systems in common. In Section 5, the two dominant modes of the EPPs and their conceptual models are concluded. A summary and discussion of the obtained results are provided in Section 6. This study will offer forecasters some useful knowledge in understanding extreme precipitation occurred over the WSB.

\section{Data and Analysis Methods}

A dataset including 2,480 stations for daily precipitation data is provided by the National Meteorological Information Centre, China Meteorological Administration (CMA). The daily reanalysis data are provided by the National Centers for Environmental Prediction and National Center for Atmospheric Research (NCEP/NCAR) with a horizontal resolution

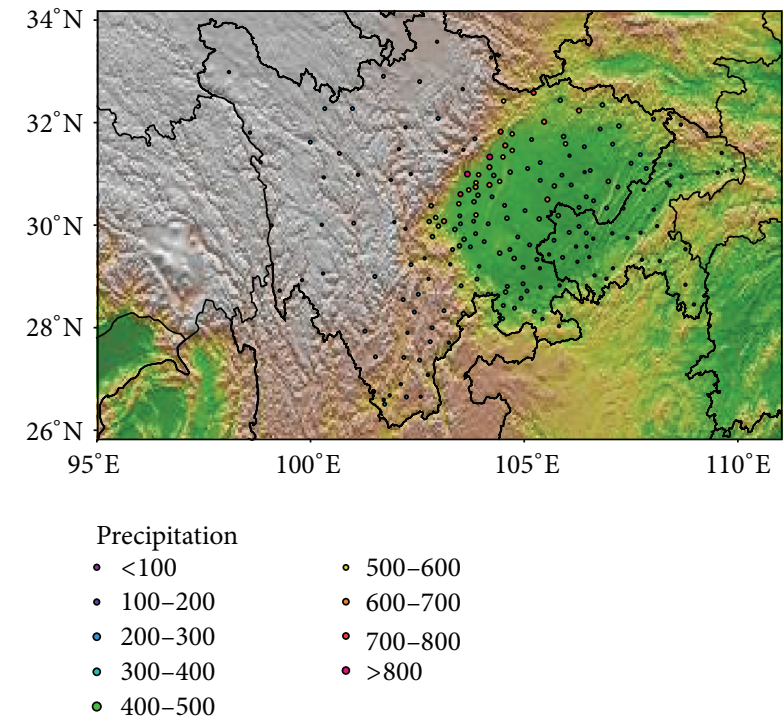

FIGURE 1: Rainfall spatial distributions during the period June 18July 19, 2013 (unit: mm).

of $2.5^{\circ} \times 2.5^{\circ}$ and 17 vertical levels [28]. To handle the complexity of circulation features of all the extreme precipitation processes, the paper computed the correlation coefficient between precipitation and the $500-\mathrm{hPa}$ geopotential height field, and then rainfall and 7 high-correlated circulation indices were dealt with the multivariable empirical orthogonal function (MV-EOF) technique to extract the dominant circulation modes of EPPs.

We also introduced several other indices, including the Balkhash Lake Depression (BalkhaD), the Baikal Blocking High (BaikaBH), the Okhotsk Sea Depression (OkhotSD), the Continent Sector of the West Pacific Subtropical High (CSWPSH), the Indian Depression (IndiaD), the South China Sea Depression (SCSD), and the West Pacific Tropical Cyclone (WPTC), defined as the area-averaged 500$\mathrm{hPa}$ geopotential height over regions of $\left(48^{\circ}-55^{\circ} \mathrm{N}, 78^{\circ}-\right.$ $\left.85^{\circ} \mathrm{E}\right),\left(48^{\circ}-58^{\circ} \mathrm{N}, 98^{\circ}-108^{\circ} \mathrm{E}\right),\left(47^{\circ}-53^{\circ} \mathrm{N}, 130^{\circ}-137^{\circ} \mathrm{E}\right),\left(27^{\circ}-\right.$ $\left.32^{\circ} \mathrm{N}, 114^{\circ}-119^{\circ} \mathrm{E}\right),\left(20^{\circ}-26^{\circ} \mathrm{N}, 81^{\circ}-90^{\circ} \mathrm{E}\right),\left(12^{\circ}-20^{\circ} \mathrm{N}, 108^{\circ}-\right.$ $\left.117^{\circ} \mathrm{E}\right)$, and $\left(14^{\circ}-20^{\circ} \mathrm{N}, 128^{\circ}-136^{\circ} \mathrm{E}\right)$, respectively. The standard deviation was denoted by $\sigma$ in this study.

\section{Overviews of the Extreme Precipitation Events in 2013}

From mid-June to mid-July in 2013, precipitation mainly concentrated over the WSB with frequent process, extreme intensity, and long duration, which resulted in multiple floods, especially in the western region. Maximum daily rainfall exceeded $800 \mathrm{~mm}$, which was remarkably above normal (Figure 1). On regional average, daily precipitation was $2 \sigma$ above normal frequently. The region recorded 4 EPPs during the period June19-22, June 30-July 2, July 3-5, and July 8-11, respectively (Figure 2). In particular, during the last process, precipitation was heaviest and rainstorm duration persisted three days longer. Such extremes occurred in 


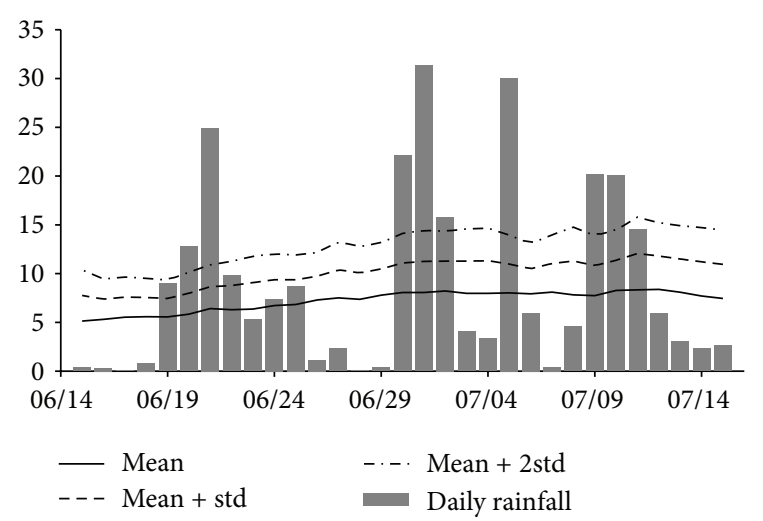

FIgURE 2: Daily regional precipitation $\left(102^{\circ}-108^{\circ} \mathrm{E}, 28^{\circ}-33^{\circ} \mathrm{N}\right)$ during the period June 15-July 15, 2013 (the bar represents daily rainfall, the solid line is daily climatical average, and the dash/dash-dot line is over onefold/twofold standard deviation above normal, resp.) (unit: $\mathrm{mm})$.

the neighborhood of 7 stations with daily rainfall over $50 \mathrm{~mm}$. That process has set the record in the Sichuan Basin since 1951 and was strictly in accordance with the regional persistent rainstorm events as defined by Chen and Zhai [29].

\section{Large-Scale Atmospheric Circulation and Water Vapor Transport Features}

The atmospheric circulation provides a basic background for understanding mechanism of extreme event. Some recent studies [30-32] indicated that in summer 2013, SSTs in the Western Pacific Warm Pool region was higher than normal. At that background, the East Asia summer monsoon is stronger than normal and the West Pacific subtropical high (WPSH) frequently strengthened and westward expanded. More than normal tropical cyclones were observed in the South China Sea. Furthermore, weaker East Asia troughs frequently occurred in the mid-high latitudes. All such circulation features are favorable for forming the "Saddle" and "Sandwich" structures thus eventually causing precipitation in WSB.

In the following, we discuss main features as shown in the 500-hPa geopotential height and water vapor transport anomaly field for the four EPPs. It is noted that the terrains of the Tibetan Plateau and the Sichuan Basin, as well as the southwest vortex and the South Asia High, also can contribute to the extreme precipitation process in WSB, which, however, are not discussed in this study.

4.1. The First Process during June 19-22 (Figure Not Shown). One ridge (over the Baikal Lake) between the double troughs (over the Balkhash Lake and the Okhotsk Sea) exhibited over the mid-high latitudes in 500-hPa geopotential height. The WPSH was larger than normal and stretched westward over the mid-lower reaches of the Yangtze River (MLRYR). Its continental sector formed an anomalous anticyclone over the Yangtze-Huaihe Rivers region. Because of the WPSH favorable position, the westerlies trough conveyed cold-dry air from the mid-high latitudes into the Sichuan Basin. At the same time, the tropical storm "Bebinca," locating over the South China Sea (SCS), transported the warm moist airflow from the south. Therefore, the water vapor convergence over the WSB stemmed from the "Bebinca" and the WPSH.

4.2. The Second Process during June 30-July 2 (Figure 3). In the mid-high latitudes, there existed a low and a high system over the western and eastern Eurasia in the 500hPa geopotential height field, respectively. In particular, the high pressure barrier developed over the northeast side of Sichuan Basin. This held back the cold airflow into the Sichuan Basin. On the other hand, the WPSH intensified and extended westward near $110^{\circ} \mathrm{E}$ and its northwestern flank was located over the WSB. Meanwhile, the tropical cyclone (TC) "Rumbia" moved into the SCS. Both WPSH and TC transported a large amount of warm water vapor into the WSB. And the terrains of the Tibetan Plateau provided favorable conditions for the moist airflow to be lifted and to release conditional or convective instabilities. Therefore, the combination of WPSH and TC played an important role in transferring the warm moist flow.

4.3. The Third Process during July 3-5 (Figure Not Shown) - This process was different from the others, which was a widespread extreme precipitation rather than a persistent precipitation in the WSB. It was a typical large-scale frontal precipitation, especially on July 5. During the process, a deep mid-latitude trough developed over the north side of the Sichuan Basin and a blocking high located over the Okhotsk Sea. Meanwhile, the WPSH intensified and extended westward, but its ridge location was more southward than normal. At this moment, the warmer stream from the WPSH confronted the colder air from the rear of the mid-high latitudes deep trough over the Sichuan Basin. Such feature created a region scale moist airflow convergence. Because there was no depression over the SCS or Western Pacific (WP), the water vapor could not be persistently supplied to the WSB. On 6 July, the mid-high latitude circulations have rectified and the WPSH retreated eastward. Therefore, the precipitationfavorable circulation could not long-lived maintain over the Sichuan Basin.

4.4. The Fourth Process during July 8-11 (Figure 4). The precipitation during this process was heaviest and the rainstorm duration persisted more than three days in the neighborhood of 7 stations with daily rainfall over $50 \mathrm{~mm}$. However, the high latitude circulation feature was seemingly opposite to the first precipitation process. The trough and ridge were active in the westerlies zone. The WPSH intensified and its continental sector extended westward near $105^{\circ} \mathrm{E}$. Moreover, the tropical storm "Soulik" made the WPSH more northward of normal. The persistent extreme precipitation process owned to the convergence between the cold airflow behind the blocking high in Baikal Lake and the warm southeasterly airflow from the westward-extending and northward-shifting WPSH over MLRYR.

In summary, four extreme precipitation processes happened over the WSB in summer 2013, including three persistent EPPs. The impacting large-scale atmospheric circulation 

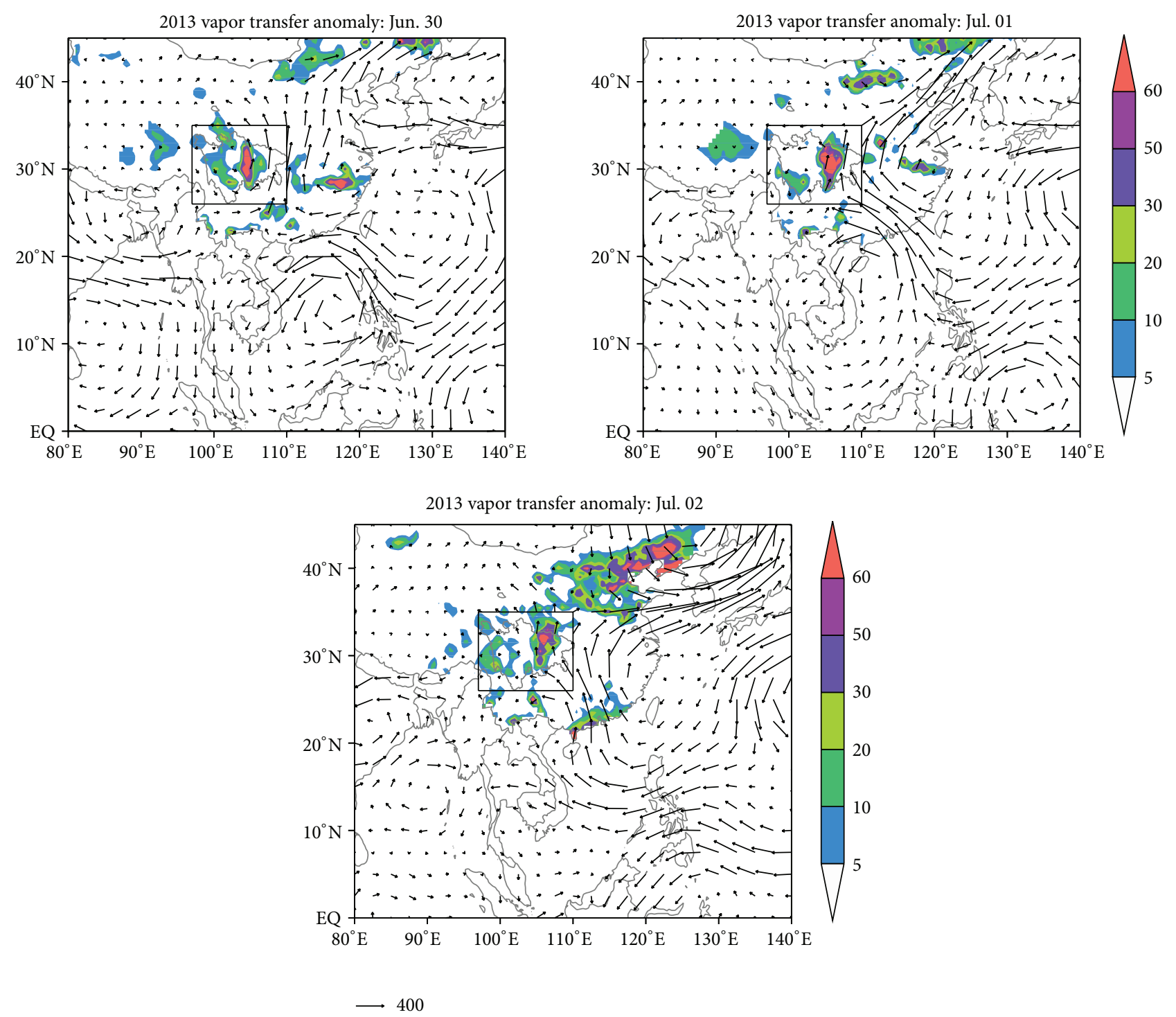

FIGURE 3: Vertically integrated water vapor transport anomaly $\left(\mathrm{kg} \cdot \mathrm{m}^{-1} \cdot \mathrm{s}^{-1}\right.$ ) (shading: rainfall $>5 \mathrm{~mm}$ ) for the 2nd process during the period June 30-July 2, 2013.

and water vapor transport features are very complicated. The EPPs tend to happen over the WSB under the following two circumstances: (a) one ridge between the double troughs (the 1st process) or a low/high value system in the western/eastern Eurasian (the 2nd process) exhibits over the midhigh latitudes. This feature combines the occurrences of a TC and the strengthening and westward-extending WPSH. (b) A blocking high develops in the high latitudes (the 3rd process) or a deep trough occurs in the westerlies zone (the 4 th process). Such circulation cooperates with an anomalous anticyclone (the 3rd and 4th processes) over SCS and the strengthening, westward-extending, and northward-shifting WPSH. Therefore, both the WPSH and the TC contribute to four EPPs over the WSB in summer 2013. Chen et al. [27] draw a similar conclusion in Sichuan on July 26-30, 2001, July 30-August 1, 2002, and September 23-26, 2008. Still in another condition, the above mentioned two types of opposite circulation pattern-an anomalous cyclone (the 1st and 2nd processes) and an anomalous anticyclones (the 3rd and 4th processes) over SCS seem to be both favorable for the rainstorm process in the WSB. But how can such contradictory conditions lead to the EPPs?

\section{Dominant Impacting System Pattern Configurations of EPPs in the WSB}

The atmospheric circulation systems, which lead to four extreme precipitation processes in the WSB in summer 2013, are too complicated to capture their similarities. To solve this difficulty, correlation coefficients between daily precipitation over the WSB and 500-hPa geopotential height field were computed from June 18 to July 20, 2013 (Figure 5). Seven high correlated key circulation systems are identified, including 

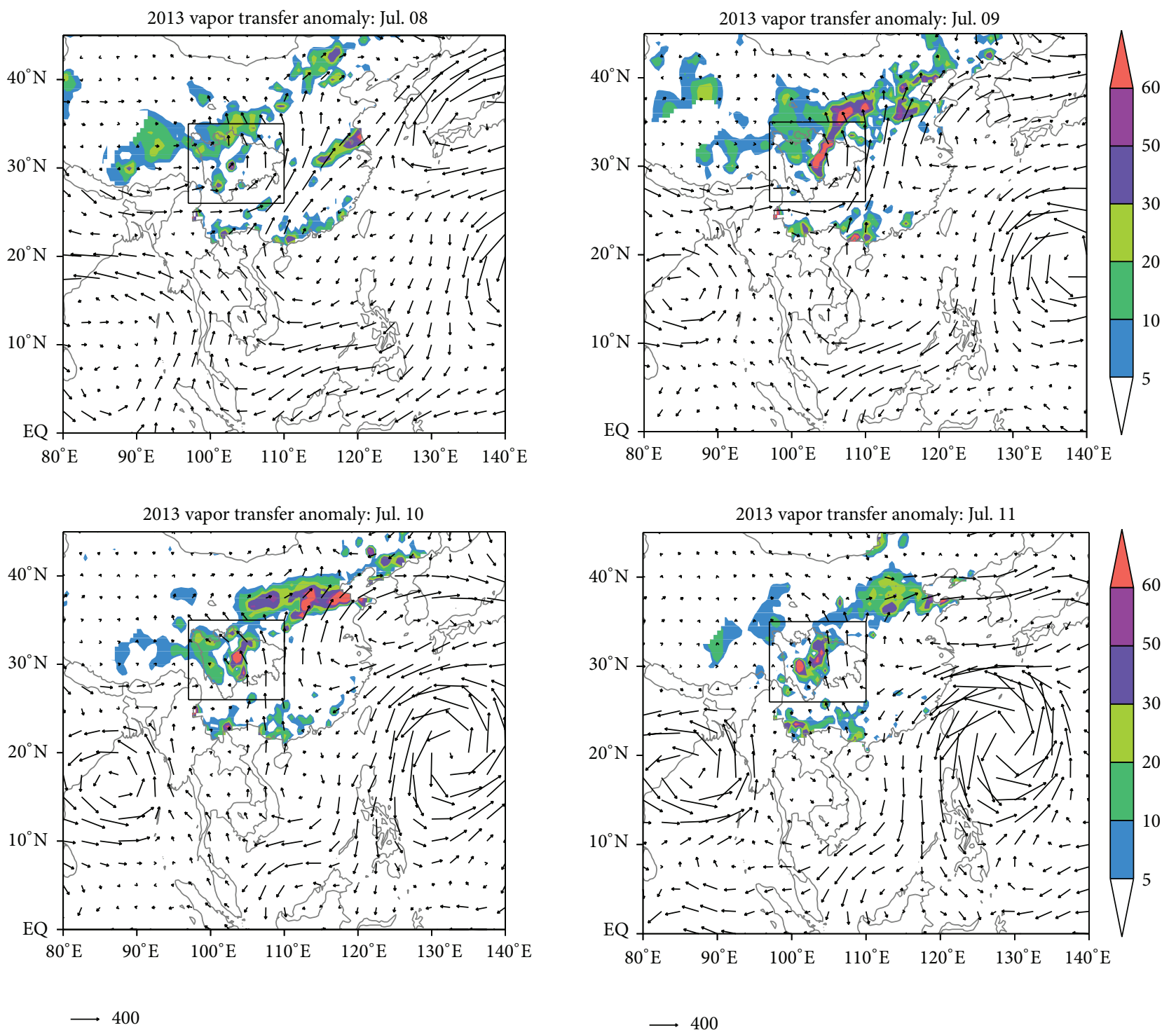

FIGURE 4: Same as in Figure 3, but for the 4th process during the period July 8-11, 2013.

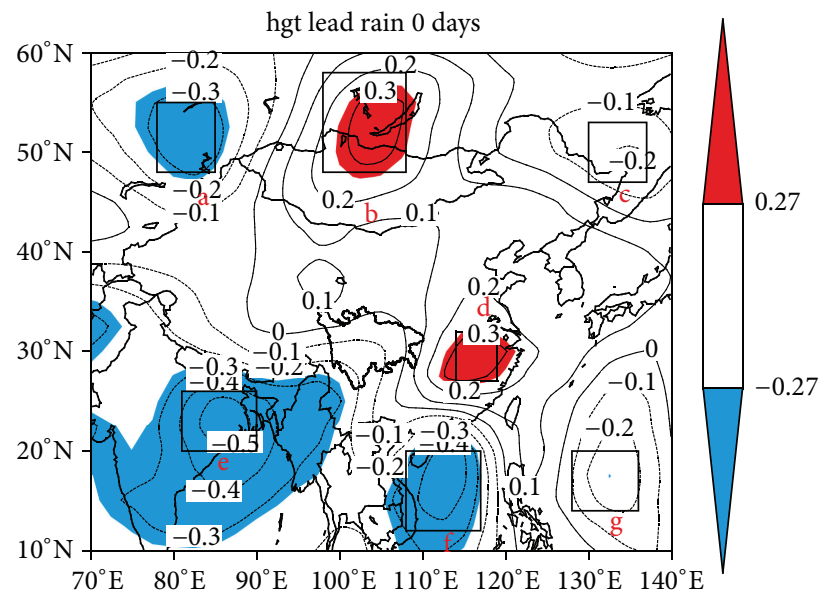

FIGURE 5: Correlation pattern between the WSB precipitation and the 500-hPa geopotential height field. The shaded regions denote correlation coefficients exceeding the $95 \%$ confidence level on the basis of Student's $t$-test. 


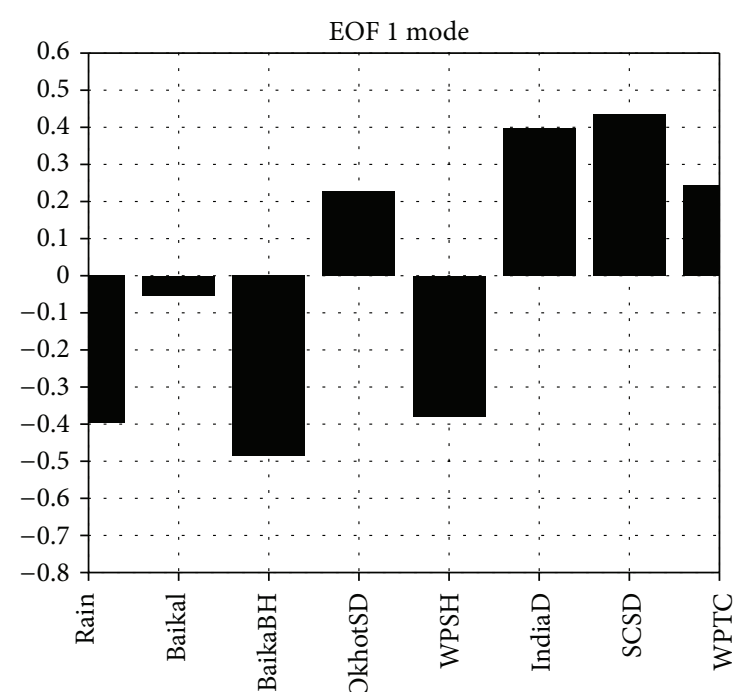

(a)

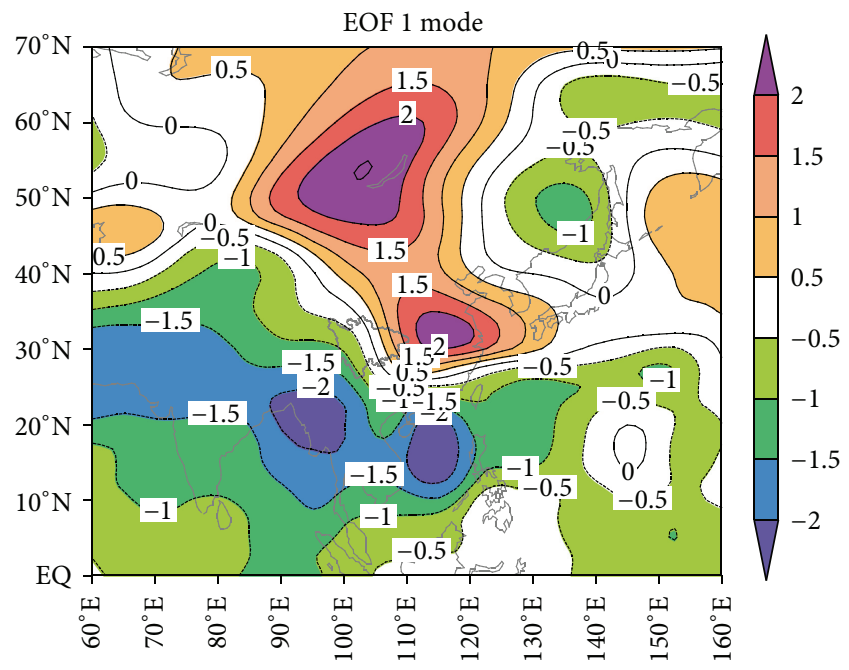

(c)

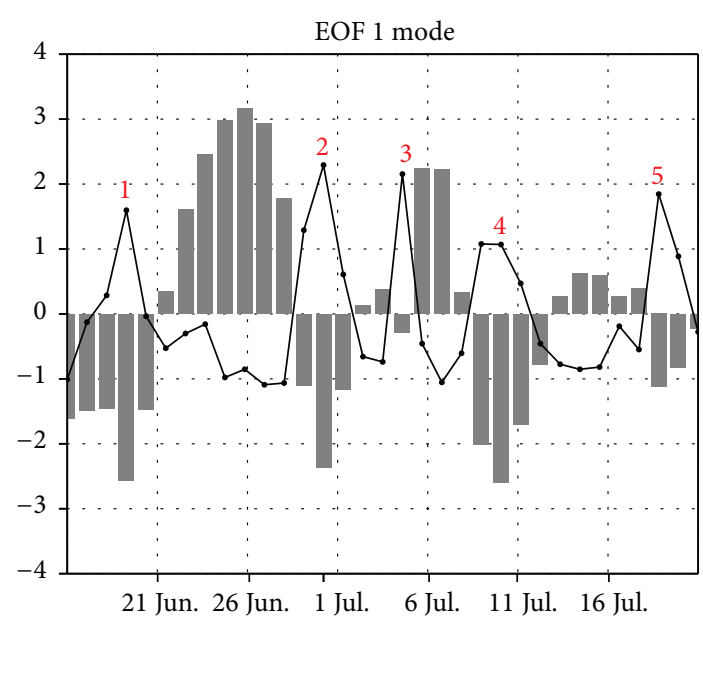

(b)

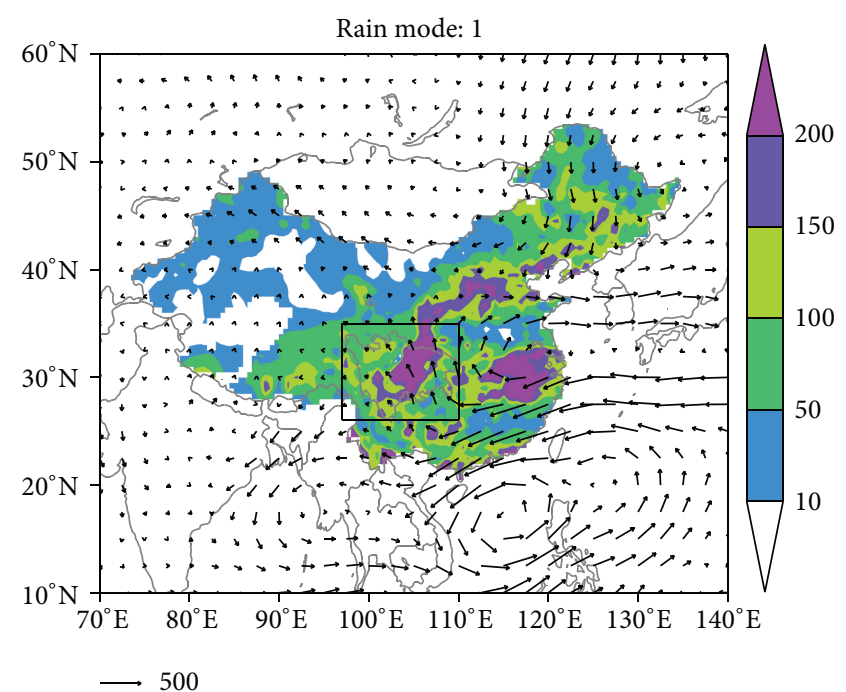

(d)

Figure 6: (a) Spatial pattern (the bar) and (b) time series (the bar) of the first mode of the MV-EOF analysis (the solid line denotes the normalized daily precipitation). (c) Composite of 500-hPa normalized geopotential height anomaly. (d) Composite of vertically integrated water vapor transport anomaly $\left(\mathrm{kg} \cdot \mathrm{m}^{-1} \cdot \mathrm{s}^{-1}\right)$ (shading: rainfall $>10 \mathrm{~mm}$ ).

(a) BalkhaD, (b) BaikaBH, (c) OkhotSD, (d) CSWPSH, (e) IndiaD, (f) SCSD, and (g) WPTC. The multiple linear regression equation between daily precipitation and the seven key factors exceeded the $95 \%$ confidence level on the basis of Student's $t$-test with multiple-correlation coefficient reaching $0.45 .35 .1 \%$ of the daily precipitation variance can be explained by all the factors, among which the BalkhaD, CSWPSH, and SCSD are more significant than others.

Secondly, daily rainfall and the aforementioned 7 highcorrelated circulation indices are dealt with by the MV-EOF analysis. Figures 6(a), 6(b), 7(a), and 7(b) depict the first two leading modes and the corresponding principal components (PCs). They account for $35.5 \%$ and $18.3 \%$ of the total variance, respectively, with a sum of $53.8 \%$. According to the rule given by North et al. [33], the first and the second modes are statistically distinguished from each other. Therefore, the first two leading modes and their corresponding atmospheric circulations are further analyzed so as to draw a common conclusion for the four EPPs.

5.1. The First Mode. Figures 6(a) and 6(b) show the spatial pattern and $\mathrm{PC}$ of the MV-EOF first mode. The main factors are BaikaBH, CSWPSH, IndiaD, and SCSD, which act on the first, second, and fourth EPPs. And the PC shows the three precipitation processes are persistent. Figures $6(\mathrm{c})$ and $6(\mathrm{~d})$ present the composite of 500-hPa normalized geopotential height and the vertically integrated water vapor transport anomaly with PC value $>1 \sigma$. In 500-hPa geopotential height field, the anomaly pattern is characterized by a "saddle" field (Figure 6(c)). It is concluded that the most importance is that 


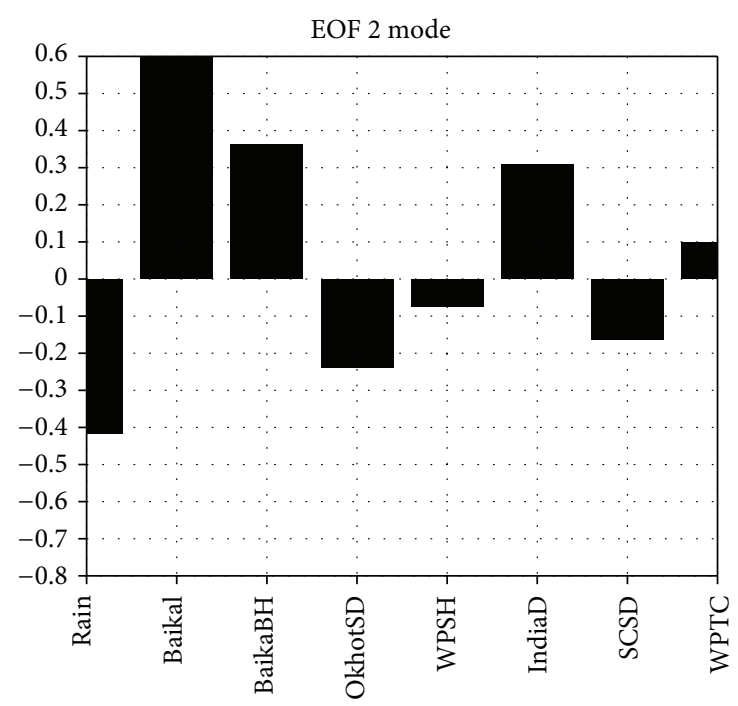

(a)

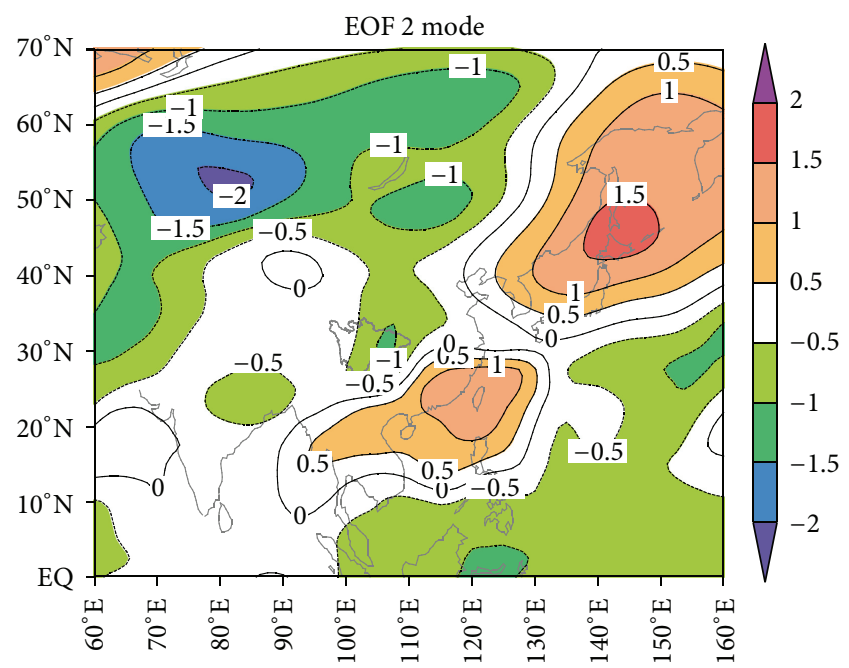

(c)

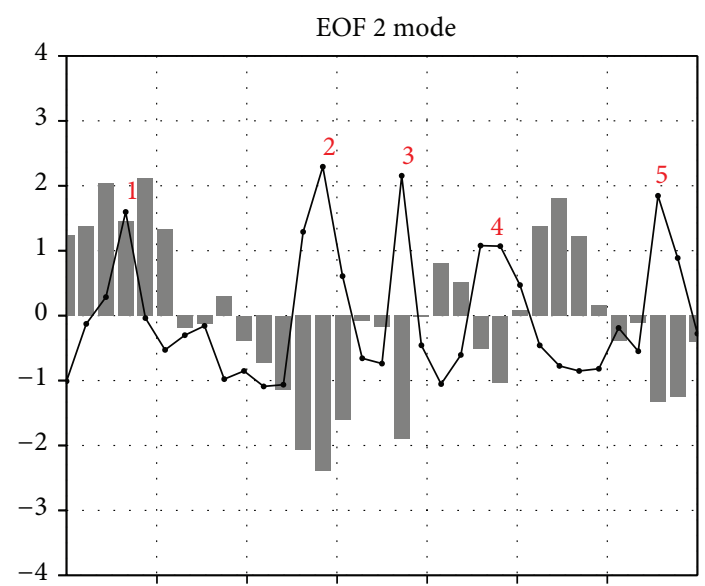

21 Jun. 26 Jun. 1 Jul. 6 Jul. 11 Jul. 16 Jul.

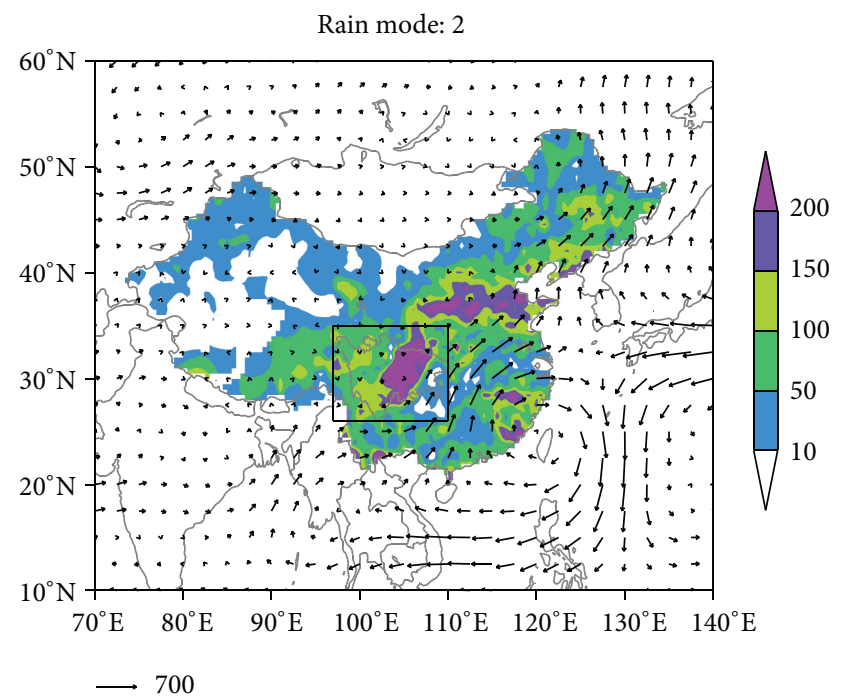

(d)

FIgURE 7: Same as in Figure 6, but for the second mode of the MV-EOF analysis.

circulation systems should match up with each other for EPPs over the WSB. When a blocking high occurring over Baikal Lake in the high latitudes, the WPSH strengthening and extending westward over MLRYR, an immense depression maintaining over the tropic region and a TC controlling over the SCS, the EPPs prefer to occur. The cold and warm airflow confront from the eastside of Sichuan Basin. This kind of circulation pattern could maintain for several days so as to produce a persistent precipitation. And this is consistent with Chen et al.s [27] previous studies according to statistics of 22 typical summer rainstorm events in Sichuan from 1981 to 2000 .

5.2. The Second Mode. Figures $7(\mathrm{a})$ and $7(\mathrm{~b})$ show the spatial pattern and PC of the second mode. It is different from the first one. The key factors are the Balkhash Lake depression, the Baikal Lake depression, the Okhotsk Sea blocking high, and the Indian depression, as well as the conjunction of the SCS anticyclone and the WPSH. The circulation configuration is in favor of the second, third, and fourth precipitation processes. The composite spatial pattern of $500-\mathrm{hPa}$ normalized geopotential height is characterized by a "Sandwich" structure (Figure 7(c)). All the anomalous circulation systems present southwest-northeast oriented distribution pattern. Two low systems inhabit from the Ural Mountain to the Baikal Lake in high latitudes and from the southern SCS to the WP in the tropics. Nevertheless, there is a high one over the northern SCS and South China, through Japan up to the Okhotsk Sea. A TC in WP pushes the WPSH westward into the SCS. Furthermore, the SCS anomalous anticyclone also attracts the WPSH westward-extending and southward-shifting so as to the two high systems form 


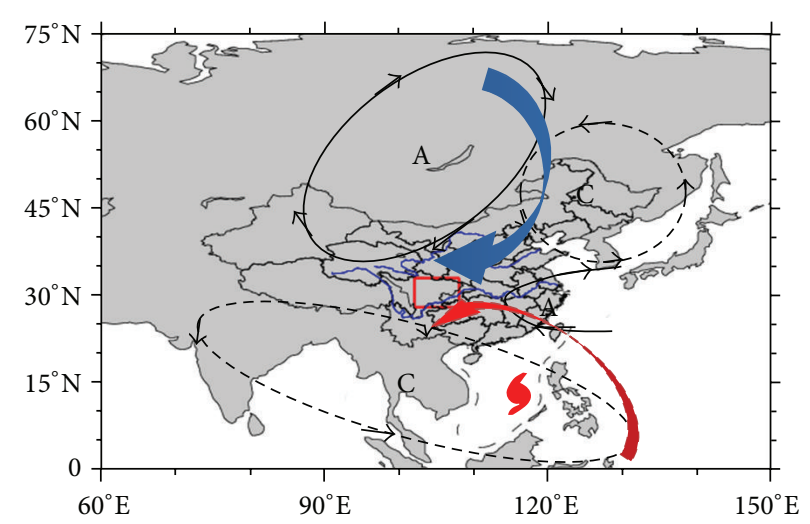

(a)

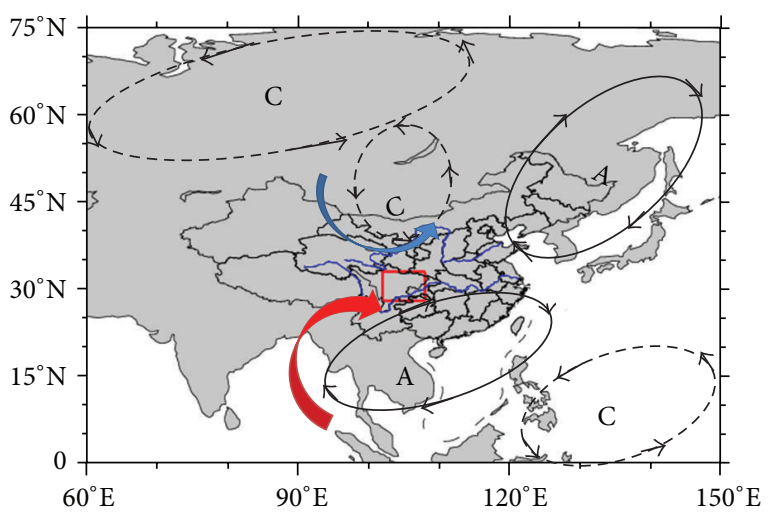

(b)

FIGURE 8: The conceptual model for the first (a) and second (b) mode of the MV-EOF analysis. The letters "A" and "C" represent anticyclone and cyclone and the red and blue arrows are warm and cold airflow, respectively.

a winning combination. Therefore, the warm stream comes from the west side of the conjunction. And the cold airflow is from the small trough over the north side of Sichuan Basin. This kind of circulation pattern is so steady that it could persist for a few days.

As can be seen in Figures 8(a) and 8(b), the conceptual models from the MV-EOF first and second mode are shown. In comparison, the WPSH is the same system in the midlatitude, but it plays a different role in these two modes. It depends on whether a depression is over SCS or WP. For the first mode, a TC over the SCS converts into the inverted trough and steers the warm airflow northward into the WSB. Locating over the MLRYR, the WPSH makes vapor convergence zone westward and conveys southeastward warm current. In the second mode, the WPSH is pushed westward by a TC in WP. Moreover, the anomalous anticyclone over the SCS attracts the WPSH westward-shifting so as to the two high value systems combine a conjunction belt. Such conjunction belt adverts southwestward warm moist airflow to merge with the cold current from the trough in the midhigh latitude. Therefore, not only do the precipitation events over the WSB depend on a blocking and/or a trough in the high latitude, but also rely on the WPSH in the mid-latitude and a TC from the tropics. The occurrence of EPPs should depend on the circulation systems matching up with each other $[24,29,34]$.

\section{Summary and Discussion}

From mid-June to mid-July in 2013, four EPPs occur during the period June 19-22, June 30-July 2, July 3-5, and July 8-11 over the WSB, respectively. Daily precipitation totals are occasionally $2 \sigma$ above normal. Through daily largescale circulation analysis, the results underscore that EPPs happen under the different steady circulation configurations. The WPSH and TC play crucial roles in EPPs over the WSB. Using MV-EOF technique, the first two leading modes are characterized by a "saddle" and a "Sandwich" pattern with accounting for $35.5 \%$ and $18.3 \%$ of the total variance, respectively. As a result, we draw the following conclusions.
(1) Both TC and WPSH play a different role in the two modes by MV-EOF. In the first mode, TC over the SCS converts into the inverted trough and steers the warm airflow northward into the WSB. Locating over the MLRYR, the WPSH make vapor convergence zone westward and convey southeastward warm airflow. In the second mode, a TC in WP pushes the WPSH westward-extending. Simultaneously, an anomalous anticyclone over the SCS attracts the WPSH. Consequently, the WPSH and the SCS anomalous anticyclone form a conjunction belt, which advects the southwesterly warm moist airflow to merge with the cold current over the WSB.

(2) Not only do the precipitation events over the WSB depend on a blocking and/or a trough in high latitude, but also rely on the WPSH in mid-latitude and a TC in the tropics. The EPPs events depend on the circulation systems matching up with each other. Especially, "Saddle" and "Sandwich" patterns are favorable for EPPs over WSB in summer 2013.

(3) Four EPPs include three persistent EPPs. The persistence of EPPs depends on the long-lived circulation systems steady over the suitable position. The third process is different from the others and is a typical large-scale frontal precipitation. Because there is no depression over the SCS or the WP, the water vapor could not be persistently supplied; meanwhile, the mid-high latitude circulations rectify on July 6, 2013. Therefore, the precipitation-favorable circulation could not long-lived maintain over the Sichuan Basin.

Our cases exemplify a situation that might be relevant for the 2013a case. During the 4 EPPs, the pretty clear indications is that the circulation systems should match up with each other and the persistence of EPPs depends on the long-lived large-scale circulation configuration steady over the suitable positions. Clearly, 4 cases are not sufficient to make overly broad generalizations. We hope eventually to be able to get the dominant impacting system configuration patterns of 
EPPs in WSB, based on a much larger set of cases than we have considered here. To clearly explain the cause and effect, a further study seems to be needed to perform numerical simulations with "imposed" such two modes at $500 \mathrm{hPa}$ to explore the impact on the precipitation distribution in future.

It is well known that the affecting factors of EPPs over WSB are very complicated, including the trough, the blocking, the WPSH, the TCs, the monsoon, the South Asian high, the southwest vortex, and the low-level shear line, as well as the other mesoscale synoptic systems. The Sichuan Basin and the Tibetan Plateau terrains make the precipitation processes more complex. Therefore, the persistent precipitation physics mechanisms are a challenge to deeply understand. Further investigation of the multiscale interaction among the atmospheric circulation, the external forcing role of the Tibetan Plateau terrain and the air-land-sea interaction and so on, may also be important and essential to understand the EPPs.

\section{Conflict of Interests}

The authors declare that there is no conflict of interests regarding the publication of this paper.

\section{Acknowledgments}

This paper was jointly supported by the National Basic Research and Development (973) Program of China (2012CB417205 and 2013CB430202), the National Natural Science Foundation of China (40905043 and 41375091), and the Open Program of State Key Laboratory of Severe Weather (2013LASW-B12).

\section{References}

[1] C.-S. Chen, W.-C. Chen, Y.-L. Chen, P.-L. Lin, and H.-C. Lai, "Investigation of orographic effects on two heavy rainfall events over southwestern Taiwan during the Mei-yu season," Atmospheric Research, vol. 73, no. 1-2, pp. 101-130, 2005.

[2] C.-S. Chen, Y.-L. Lin, N.-N. Hsu, C.-L. Liu, and C.-Y. Chen, "Orographic effects on localized heavy rainfall events over Southwestern Taiwan on 27 and 28 June 2008 during the postMei-Yu period," Atmospheric Research, vol. 101, no. 3, pp. 595610, 2011.

[3] C.-S. Chen, Y.-L. Lin, H.-T. Zeng, C.-Y. Chen, and C.-L. Liu, "Orographic effects on heavy rainfall events over northeastern Taiwan during the northeasterly monsoon season," Atmospheric Research, vol. 122, pp. 310-335, 2013.

[4] C. A. Doswell, C. Ramis, R. Romero, and S. Alonso, "A diagnostic study of three heavy precipitation episodes in the Western Mediterranean region," Weather and Forecasting, vol. 13, no. 1, pp. 102-124, 1998.

[5] D. A. Ahijevych, C. A. Davis, R. E. Carbone, and J. D. Tuttle, "Initiation of precipitation episodes relative to elevated terrain," Journal of the Atmospheric Sciences, vol. 61, no. 22, pp. 27632769, 2004.

[6] R. Y. Lu and H. Ye, "Decreasing trend in summer precipitation over the western Sichuan Basin since the 1950s," Atmospheric and Oceanic Science Letters, vol. 4, no. 2, pp. 114-117, 2011.

[7] S. H. Yu, J. M. Teng, and G. B. He, "The numerical experiment of plateau terrain influencing for a suddenly arising torrential rain in the west of Sichuan Basin," Scientia Atmospheric Sinica, vol. 22, no. 3, pp. 379-383, 1998.

[8] G. B. He, "Simulation of impact of steep terrain on east side of Qinghai-Xizang plateau on mesoscale vortex and rain storm over the basin," Plateau Meteorology, vol. 25, no. 3, pp. 430-441, 2006 (Chinese).

[9] C. Li, J. Chen, and G. B. He, "Impact of the steep terrain of Eastern Qinghai-Xizang Plateau on the genesis and development of extreme heavy rainfall event," Plateau Meteorology, vol. 25, no. 3, pp. 442-450, 2006 (Chinese).

[10] G. X. Wu, Y. Liu, T. Wang et al., "The influence of mechanical and thermal forcing by the Tibetan Plateau on Asian climate," Journal of Hydrometeorology, vol. 8, no. 4, pp. 770-789, 2007.

[11] A. M. Duan, G. X. Wu, Y. M. Liu, Y. M. Ma, and P. Zhao, "Weather and climate effects of the Tibetan Plateau," Advances in Atmospheric Sciences, vol. 29, no. 5, pp. 978-992, 2012.

[12] S. H. Yu, "Resultant analysis of large-Scale heavy rain storm over Sichuan Basin," Plateau Meteorology, vol. 3, no. 1, pp. 58-67, 1984 (Chinese).

[13] C. Y. Zhou, Y. Q. Li, Q. L. Bu, and J. Peng, "Features of droughtflood coexistence in West and East of Sichuan-Chongqing Basin in midsummer and its relating background of atmospheric circulation," Plateau Meteorology, vol. 30, no. 3, pp. 620-727, 2011 (Chinese).

[14] R. Shi, Y. R. Chen, and C. G. Wang, "Comparative analysis of the influence of the Subtropical high on the torrential rainfall in Sichuan Basin," Plateau and Mountain Meteorology Research, vol. 30, no. 4, pp. 18-25, 2010 (Chinese).

[15] D. Q. Hu, R. Y. Lu, Q. Su, and G. Z. Fan, "Interannual variation in the mid-summer rainfall over the western Sichuan Basin and the associated circulation anomalies," Chinese Journal of Atmospheric Sciences, vol. 38, no. 1, pp. 13-20, 2014 (Chinese).

[16] D. Chen, Y. Q. Li, and R. H. Huang, "The physical process analyses of the Southwest vortex development and its effect on heavy rainfall in Eastern Sichuan under the saddle pattern background of large-scale circulations," Chinese Journal of Atmospheric Sciences, vol. 31, no. 2, pp. 185-201, 2007 (Chinese).

[17] S. X. Zhao and S. M. Fu, "An analysis on the southwest vortex and its environment fields during heavy rainfall in eastern Sichuan Province and Chongqing in September 2004," Chinese Journal of Atmospheric Sciences, vol. 31, no. 6, pp. 1059-1074, 2007 (Chinese).

[18] C. H. Huang, G. P. Li, J. L. Niu, L. Luo, and W. Zhang, "Moist helicity analysis of a heavy rainstorm in Sichuan Basin induced by Plateau vortex moving eastward," Plateau Meteorology, vol. 30, no. 6, pp. 1427-1434, 2011 (Chinese).

[19] Y. R. Chen, Y. Q. Li, C. G. Wang, and R. Y. Deng, "Study on the relationship between South Asia High and rainfall of SichuanChongqing regions in summer," Plateau Meteorology, vol. 28, no. 3, pp. 539-548, 2009 (Chinese).

[20] Y. H. Kuo, L. S. Cheng, and R. A. Anthes, "Mesoscale analyses of the Sichuan flood catastrophe, 11-15 July 1981," Monthly Weather Review, vol. 114, no. 11, pp. 1984-2003, 1986.

[21] H. Y. Xiao and S. H. Yu, "Subtropical high activity and Sichuan rainstorm," Journal of Sichuan Meteorology, vol. 23, no. 2, pp. 212, 2003 (Chinese).

[22] Sichuan Provincial Meteorological Bureau, Handbook of ShortTerm Weather Forecasting in Sichuan Province, China Meteorological Press, Beijing, China, 2015, (Chinese).

[23] Y. F. Zhu and R. C. Yu, "Interannual variation of summer precipitation in the west of Sichuan Basin and its relationship 
with large-Scale circulation," Chinese Journal of Atmospheric Sciences, vol. 27, no. 6, pp. 1045-1056, 2003 (Chinese).

[24] Y. Chen and Y. H. Ding, "Cold air activities in July 2004 and its impacts on intense rainfalls over Southwest China," Acta Meteorologic Sinica, vol. 64, no. 6, pp. 743-759, 2006 (Chinese).

[25] B. Zhou and J. F. Wen, "Circulation of a persistent heavy rain and its diabatic heating characteristics over the North of Chongqing and the east of Sichuan in 2004," Journal of Applied Meteorological Science, vol. 17, supplement, pp. 71-78, 2006 (Chinese).

[26] Q. Y. Gu, D. X. Xiao, and C. H. Huang, “Trigger role of the lowlevel jet for the continuous rainstorm in the northwest side of subtropical high," Meteorological Monthly, vol. 35, no. 4, pp. 5967, 2009 (Chinese).

[27] D. Chen, L. Gu, and X. W. Jiang, "Characteristics of large-scale circulation background of summer heavy rainfall in Sichuan during 1981 to 2000," Transactions of Atmospheric Sciences, vol. 33, no. 4, pp. 443-450, 2010 (Chinese).

[28] E. Kalnay, M. Kanamitsu, R. Kistler et al., "The NCEP/NCAR 40-year reanalysis project," Bulletin of the American Meteorological Society, vol. 77, no. 3, pp. 437-471, 1996.

[29] Y. Chen and P. Zhai, "Persistent extreme precipitation events in China during 1951-2010," Climate Research, vol. 57, no. 2, pp. 143-155, 2013.

[30] Z. Q. Gong, Y. J. Wang, Z. Y. Wang, L. J. Ma, C. H. Sun, and S. Q. Zhang, "Briefly analysis on climate anomalies and causations in summer 2013," Meteorological Monthly, vol. 40, no. 1, pp. 119125, 2014 (Chinese).

[31] F. Zhang and L. F. He, "Analysis of the June 2013 atmospheric circulation and weather," Meteorological Monthly, vol. 39, no. 9, pp. 1227-1232, 2013 (Chinese).

[32] Y. C. Lin, J. Xu, and F. H. Zhang, "Analysis of the July 2013 atmospheric circulation and weather," Meteorological Monthly, vol. 39, no. 10, pp. 1379-1384, 2013 (Chinese).

[33] G. R. North, T. L. Bell, and R. F. Cahalan, "Sampling errors in the estimation of empirical orthogonal functions," Monthly Weather Review, vol. 110, no. 7, pp. 699-706, 1982.

[34] Y. Chen and P. M. Zhai, "Two types of typical circulation patterns for the persistent extreme precipitation in Central Eastern China," Quarterly Journal of the Royal Meteorological Society, vol. 140, no. 682, pp. 1467-1478, 2014. 

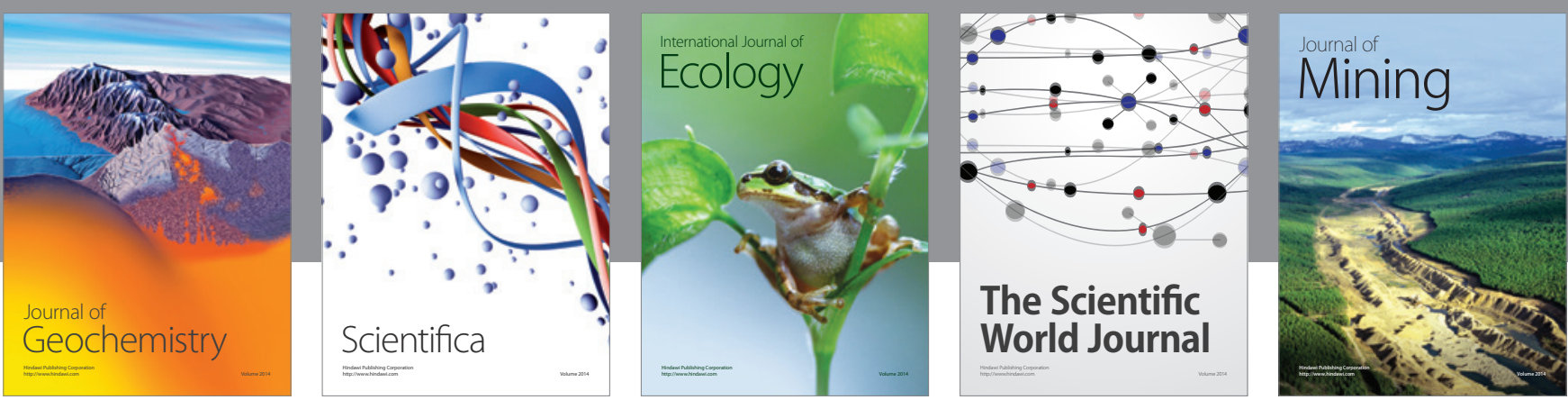

The Scientific World Journal
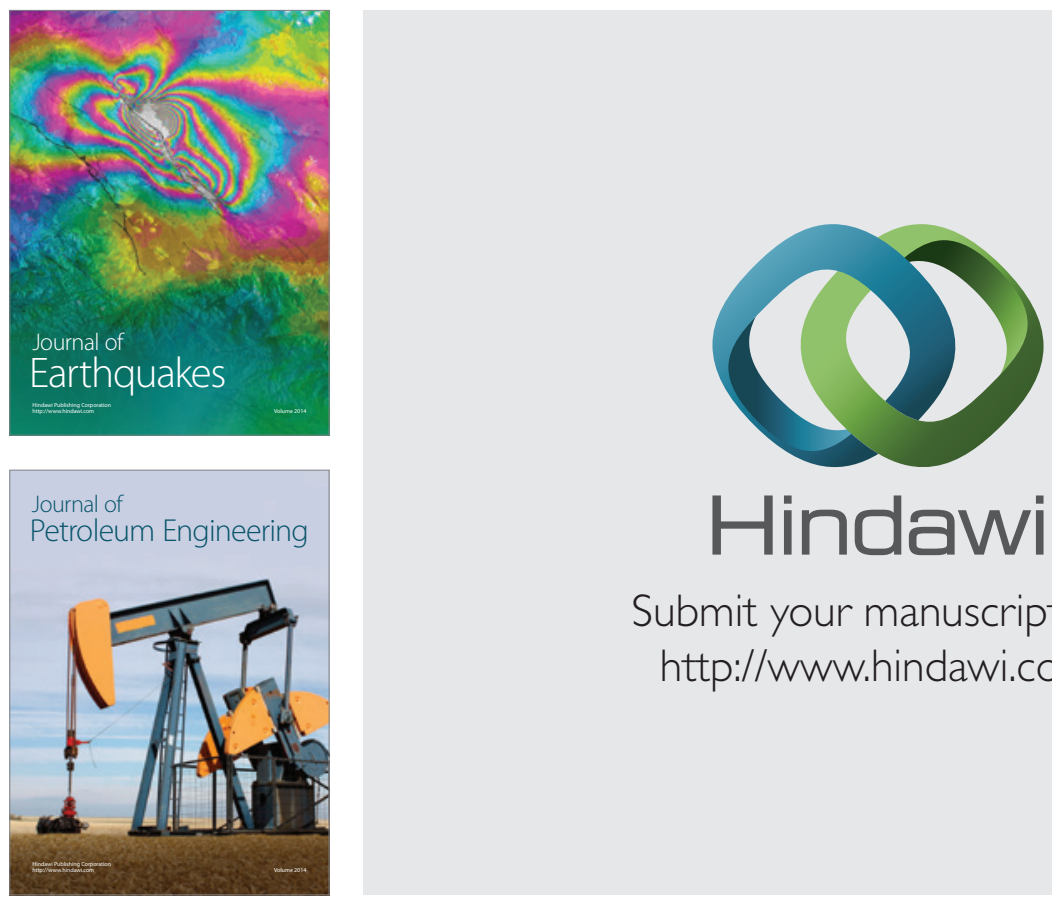

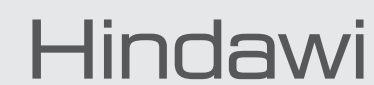

Submit your manuscripts at

http://www.hindawi.com
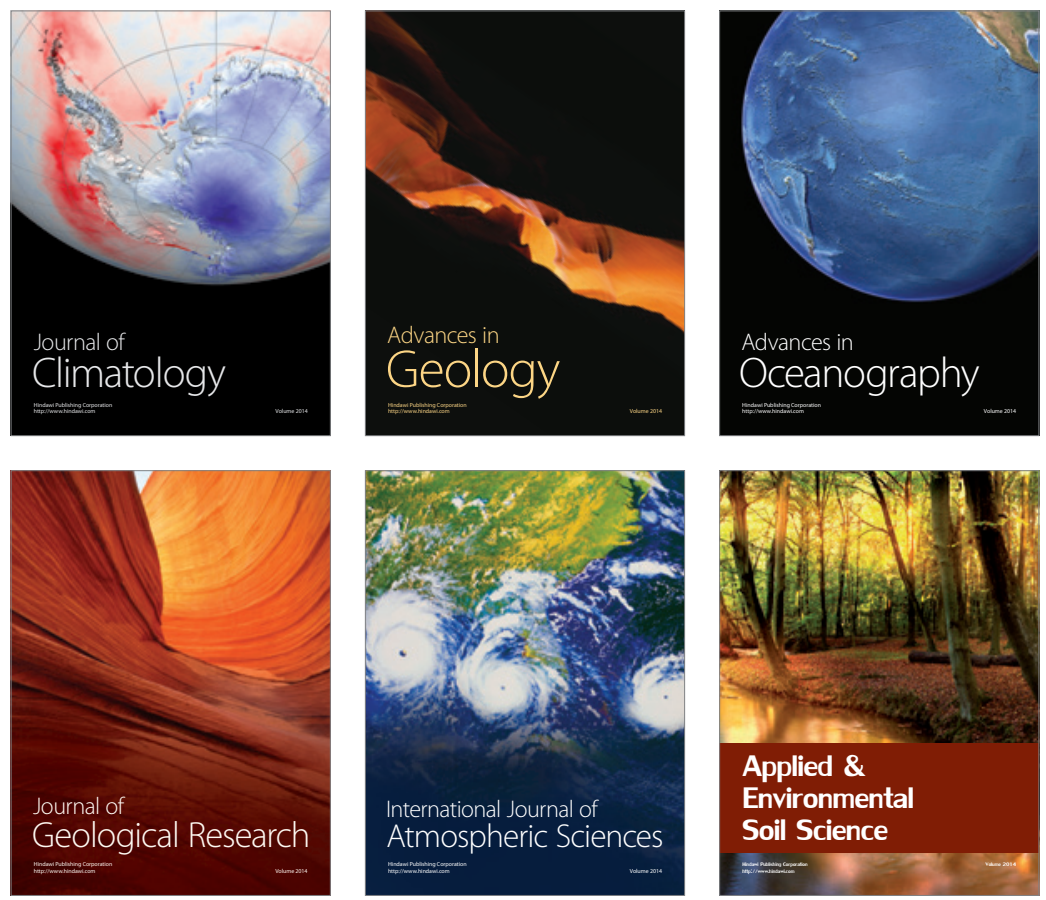
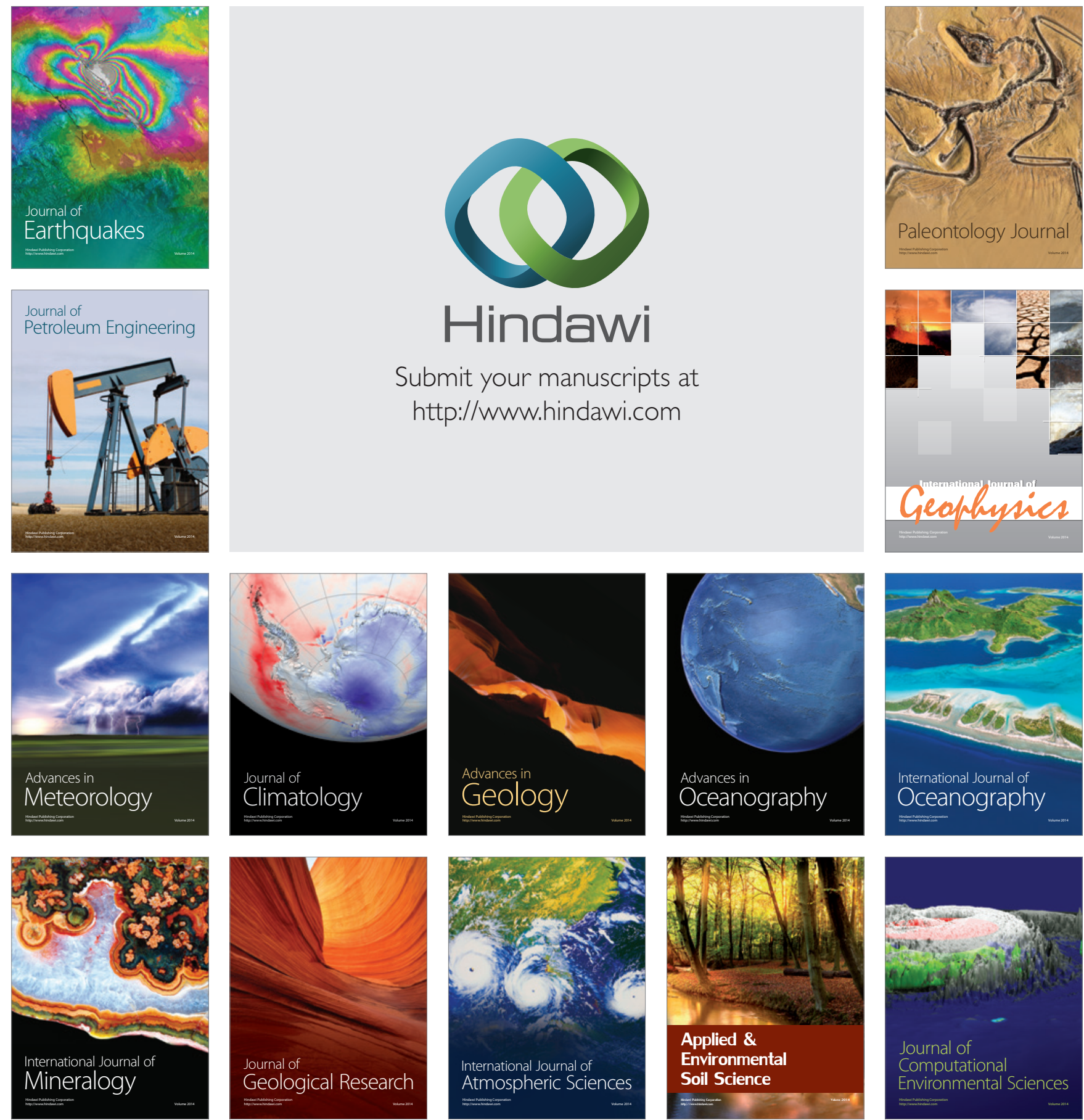